\title{
Travelling waves near a critical point of a binary fluid mixture
}

\author{
Henri Gouin \\ C.N.R.S. U.M.R. $6181 \&$ University of Aix-Marseille, \\ Case 322, Av. Escadrille Normandie-Niemen, 13397 Marseille Cedex 20 France \\ Augusto Muracchini and Tommaso Ruggeri \\ Department of Mathematics and Research Center of Applied Mathematics \\ University of Bologna, Via Saragozza 8, 40123 Bologna, Italy.
}

\begin{abstract}
Travelling waves of densities of binary fluid mixtures are investigated near a critical point. The free energy is considered in a non-local form taking account of the density gradients. The equations of motions are applied to a universal form of the free energy near critical conditions and can be integrated by a rescaling process where the binary mixture is similar to a single fluid. Nevertheless, density solution profiles obtained are not necessarily monotonic. As indicated in Appendix, the results might be extended to other topics like finance or biology.
\end{abstract}

Key words: Binary fluid mixtures; critical points; travelling waves; rescaling process.

PACS: 46.15.Cc ; 47.35.Fg ; 47.51.+a ; 64.75.Ef

\section{Introduction}

In physical chemistry, thermodynamics and condensed matter physics, a critical point specifies the conditions (temperature, pressure and concentration) at which distinct phases do not exist [6]2]29. There are multiple types of critical points such as vapor-liquid or liquid-liquid critical point. A single fluid has a

Email addresses: henri.gouin@univ-cezanne.fr (Henri Gouin), augusto.muracchini@unibo.it, tommaso.ruggeri@unibo.it (Augusto

Muracchini and Tommaso Ruggeri).

Preprint submitted to International Journal of Non-Linear Mechanics vol. 47, p.p. 77-84 (2012). 
unique critical point associated with given temperature, pressure and density. For binary mixtures of fluids, in the space of temperature, pressure, concentration, critical points are represented by a curve in a convenient domain [26]; to each temperature we can associate a critical pressure and two critical densities corresponding to the mixture components [13,36].

An important thermodynamical potential is related to the mixture volume free energy $32,33,34$. At a given temperature, the volume free energy is associated with the spinodal curve connecting the two different phases of the binary mixture. Due to conditions of equilibrium of phases, it is possible to form a general expansion of the free energy near a critical point. This form is known in the literature by means of physical chemistry considerations [37] and is the form we use in our calculations.

By calculations in molecular theories, the densities of the components fluctuate near a critical point [24]. In the following, we use a continuous model to investigate how the average variations of densities are related to molecular interactions. Two assumptions are explicit [4,18,38]:

i) The component densities are assumed to be smooth functions of the distance from an interface layer which is assumed to be flat on the scale of molecular sizes. The correlation lengths are assumed to be greater than intermolecular distances [21,23]; this is the case when at a given temperature $T$ the parameters are close to the ones of a critical state [35].

ii) The binary mixture is considered in the framework of a mean-field theory. This means, in particular, that the free energy of the mixture is a classical so-called "gradient square functional". This kind of Landau-Ginzburg model consisting of a quadratic form of the density gradients comes from Maxwell and van der Walls original ideas [27,31,42,44]. At given critical conditions, the coefficients of the quadratic form are constant.

This point of view that, in non-homogeneous regions, the mixture may be treated as bulk phase with a local free-energy density and an additional contribution arising from the non-uniformity which may be approximated by a gradient expansion truncated at the second order is most likely to be successful and perhaps even quantitatively accurate near a critical point [37]. The approximation of mean field theory does provide a good understanding and allows one to explicitly calculate the magnitude of the coefficients of the model. These non-linear equations are able to represent interface layer and bulks and consequently allow to build a complete theory of the mixtures in non-homogeneous domains in dynamics.

In Section 2 we recall the equations of motion in a pure mechanical process obtained through the Hamilton variational principle.

Section 3 is devoted to travelling waves without dissipation. Due to the fact that the equations are Galilean invariant, the case of equilibrium and the case 
of motion are analyzed together.

In Section 4 by means of a rescaling process taking the vicinity of a critical point into account, we integrate the equation for equilibrium as well as for motions with dissipation.

Two appendices present the motion equations and the mathematical reason of the choice of the free energy form near a critical point of a binary mixture of fluids obtained by a new method issued from differential geometry.

\section{Isothermal motion of a binary fluid mixture near a critical point}

We study a mixture of two fluids by a mechanical process. No assumption has to be done about composition or miscibility. The motion of a two-fluid continuum can be represented with two surjective differentiable mappings (see Fig. 1) [1,15,16,22]:

$$
\mathbf{z} \rightarrow \mathbf{X}_{1}=\Phi_{1}(\mathbf{z}) \text { and } \mathbf{z} \rightarrow \mathbf{X}_{2}=\Phi_{2}(\mathbf{z})
$$

where subscripts 1 and 2 are associated with each constituent of the mixture. Term $\mathbf{z}=(t, \mathbf{x})$ denotes Euler variables in space-time $\mathcal{W}$ and terms $\mathbf{X}_{1}$ and $\mathbf{X}_{2}$ denote the Lagrange variables of constituents in reference spaces $\mathcal{D}_{o 1}$ and $\mathcal{D}_{o 2}$ respectively.

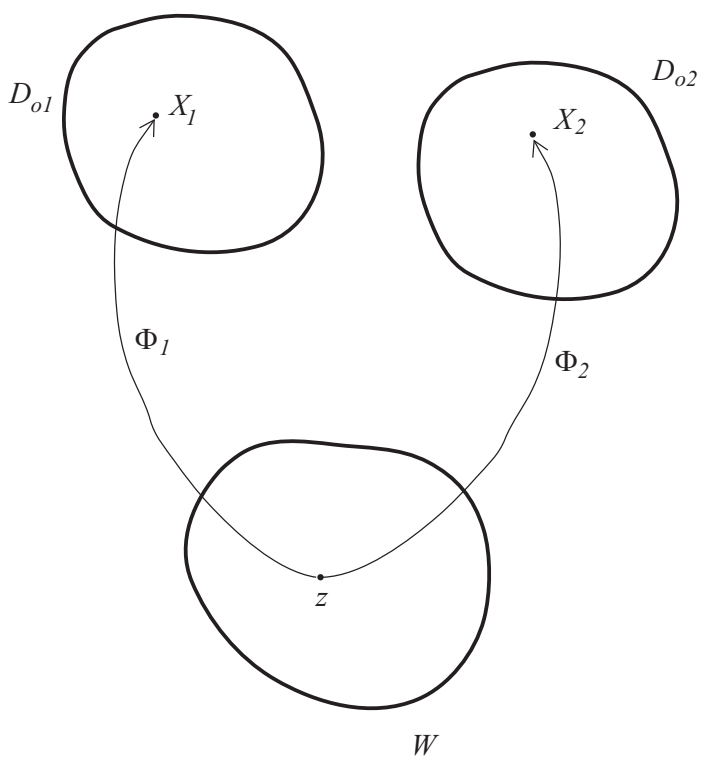

Fig. 1. General representation of a two-fluid continuum motion. 
In the pure mechanical case, the Lagrangian density of the mixture is

$$
L=\frac{1}{2} \rho_{1} \mathbf{v}_{1}^{2}+\frac{1}{2} \rho_{2} \mathbf{v}_{2}^{2}-e-\rho_{1} \Omega_{1}-\rho_{2} \Omega_{2},
$$

where $\mathbf{v}_{1}$ and $\mathbf{v}_{2}$ denote the velocity vectors of each constituent, $\rho_{1}$ and $\rho_{2}$ are the densities, $\Omega_{1}$ and $\Omega_{2}$ are the external force potentials depending only on $\mathbf{z}=(t, \mathbf{x})$ and $e$ is the volume energy [2,14].

The expression of the Lagrangian is in a general form. In fact dissipative phenomena imply that $\mathbf{v}_{1}$ is almost equal to $\mathbf{v}_{2}$; it is the reason why we do not take account of some kinetic energy associated with the relative velocity of the components which is of smaller order (at least of order 2) and will be negligible in travelling wave behavior [5|25/28]. Because of the interaction between the constituents, the volume energy $e$ is not the sum of the energies of each constituent of the mixture, like for Euler mixtures of fluids. The mixture is assumed not to be chemically reacting. Conservations of masses require

$$
\rho_{i} \operatorname{det} \mathbf{F}_{i}=\rho_{o i}\left(\mathbf{X}_{i}\right)
$$

where subscript $i$ belongs to $\{1,2\}$. At $t$ fixed, the deformation gradient $\partial \mathbf{x}_{i} / \partial \mathbf{X}_{i}$ associated with $\Phi_{i}$ is denoted by $\mathbf{F}_{i}$ and $\rho_{o i}$ is the reference specific mass in $\mathcal{D}_{o i}$.

Eqs (1) are equivalent to the Eulerian form

$$
\frac{\partial \rho_{i}}{\partial t}+\operatorname{div} \rho_{i} \mathbf{v}_{i}=0
$$

The volume energy $e$ is given by the behavior of the mixture [7,11,12]. In our mechanical case, for an energy depending on gradients of densities, the volume energy is

The potential

$$
e=e\left(\rho_{1}, \rho_{2}, \operatorname{grad} \rho_{1}, \operatorname{grad} \rho_{2}\right)
$$

$$
\mu_{i}=\frac{\partial e}{\partial \rho_{i}}-\frac{\partial}{\partial x_{\gamma}}\left(\frac{\partial e}{\partial \rho_{i, \gamma}}\right)
$$

defines the specific free enthalpy or chemical potential of the constituent $i$ of the mixture [16]. Subscript $\gamma$ corresponds to the spatial derivatives associated with gradient terms. Usually, summation is made on repeated subscript $\gamma$. In practice, we consider a quadratic form with constant coefficients $C_{1}, C_{2}, D$

$$
Q=C_{1}\left(\operatorname{grad} \rho_{1}\right)^{2}+2 D \operatorname{grad} \rho_{1} \operatorname{grad} \rho_{2}+C_{2}\left(\operatorname{grad} \rho_{2}\right)^{2}
$$

such that

$$
e=g_{o}\left(\rho_{1}, \rho_{2}\right)+\frac{1}{2} Q
$$

where $g_{o}\left(\rho_{1}, \rho_{2}\right)$ is the value of the volume energy of the homogeneous bulks.

To obtain the equations of motions, we use a variational principle whose original feature is to choice variations in reference spaces (Fig. 1). They are as- 
sociated with a two-parameter family of virtual motions of the mixture (see Appendix A).

The equation of the motion of each constituent of the mixture writes ([19] and the references therein)

$$
\mathbf{a}_{i}+\operatorname{grad}\left(\mu_{i}+\Omega_{i}\right)=0, \quad i=\{1,2\}
$$

where $\mathbf{a}_{i}$ denotes the acceleration of the component $i(i=1,2)$. In applications, the motions are supposed to be isothermal ( $T$ denotes the common temperature value of the two components) and correspond to strong heat exchange between components. In thermodynamics, this case corresponds to a function $g_{o}\left(\rho_{1}, \rho_{2}\right)$ as the volume free energy of the homogeneous mixture at temperature $T$.

In our model, the equations of motion (44) yield

$$
\left\{\begin{array}{l}
\mathbf{a}_{1}=\operatorname{grad}\left\{C_{1} \Delta \rho_{1}+D \Delta \rho_{2}-g_{o, \rho_{1}}\right\} \\
\mathbf{a}_{2}=\operatorname{grad}\left\{D \Delta \rho_{1}+C_{2} \Delta \rho_{2}-g_{o, \rho_{2}}\right\}
\end{array}\right.
$$

Taking Eq. (2) into account, we can note that the two equations of system (4) are equivalent to the system

$$
\frac{\partial \rho_{i} \mathbf{v}_{i}}{\partial t}+\operatorname{div}\left(\rho_{i} \mathbf{v}_{i} \otimes \mathbf{v}_{i}\right)+\operatorname{grad}\left(\rho_{i} \mu_{i}\right)=\mu_{i} \operatorname{grad} \rho_{i}, \quad i=\{1,2\}
$$

for which equations of components are not in divergence form, but the summation of the two equations and the fact that $\sum_{i=1}^{2} \mu_{i} \operatorname{grad} \rho_{i}=\operatorname{grad} e$ allow to obtain the total motion of the mixture in divergence form [16]19]. Therefore, while the global momentum equation represents a balance law, individually, the equations of system (2) do not.

\section{Travelling waves for an isothermal fluid mixture in one-dimensional case}

\subsection{The system of equations for travelling waves}

An interface in a two-phase mixture is generally schematized by a surface without thickness. Far from critical conditions, this layer is of nanometre size and density and entropy gradients are very large. This is not the case in the immediate vicinity of critical points where fluctuations of densities strongly diverge. Our goal is to schematize the average of these fluctuations by means of travelling waves in a continuous model using an energy in form (3). 
To obtain one-dimensional travelling waves in isothermal case when we neglect diffusion and viscosity, we are looking for solutions only depending on the variable [3, 17,20,43]

$$
\xi=x-s t
$$

where $s$ denotes the wave velocity. Equations (2) of conservation of masses write

$$
\frac{\partial \rho_{i}}{\partial t}+\frac{\partial\left(\rho_{i} v_{i}\right)}{\partial x}=0
$$

where $v_{i}$ denotes the one-dimensional velocity of component $i$. Equivalently 41],

$$
\frac{d}{d \xi}\left\{\rho_{i}\left(v_{i}-s\right)\right\}=0 \quad \Longleftrightarrow \quad v_{i}=\frac{d_{i}}{\rho_{i}}+s,
$$

where $d_{i}, i=\{1,2\}$, are constant along the motion and have the dimension of a mass flow. The acceleration of mixture component $i$ is

$a_{i}=\frac{\partial v_{i}}{\partial x} v_{i}+\frac{\partial v_{i}}{\partial t} \quad \Longleftrightarrow \quad a_{i}=\frac{d}{d \xi}\left\{\frac{\left(v_{i}-s\right)^{2}}{2}\right\} \quad \Longleftrightarrow \quad a_{i}=\frac{1}{2} \frac{d}{d x}\left(\frac{d_{i}^{2}}{\rho_{i}^{2}}\right)$

and system (5) yields

$$
\begin{cases}C_{1} \rho_{1}^{\prime \prime}+D \rho_{2}^{\prime \prime}=\mu_{1}+\frac{1}{2} \frac{d_{1}^{2}}{\rho_{1}^{2}}+k_{1} & \\ D \rho_{1}^{\prime \prime}+C_{2} \rho_{2}^{\prime \prime}=\mu_{2}+\frac{1}{2} \frac{d_{2}^{2}}{\rho_{2}^{2}}+k_{2} & \text { or } \quad \mathbf{R X}^{\prime \prime}=\left(\frac{\partial Y}{\partial \mathbf{X}}\right)^{T},\end{cases}
$$

where $k_{1}$ and $k_{2}$ are two constants, superscript ${ }^{T}$ denotes the transposition and

$\mathbf{X}=\left(\begin{array}{c}\rho_{1} \\ \rho_{2}\end{array}\right), \mathbf{R}=\left(\begin{array}{cc}C_{1} & D \\ D & C_{2}\end{array}\right), Y\left(\rho_{1}, \rho_{2}\right)=g_{o}\left(\rho_{1}, \rho_{2}\right)-\frac{1}{2} \frac{d_{1}^{2}}{\rho_{1}}-\frac{1}{2} \frac{d_{2}^{2}}{\rho_{2}}+k_{1} \rho_{1}+k_{2} \rho_{2}$

Here ${ }^{\prime}=d / d \xi$ denotes the derivation along the ray. Let us note that the sum of equations of system (7), respectively multiplied by $\rho_{1}^{\prime}$ and $\rho_{2}^{\prime}$, yields the first integral

$g_{o}\left(\rho_{1}, \rho_{2}\right)+k_{1} \rho_{1}+k_{2} \rho_{2}-\frac{1}{2} \frac{d_{1}^{2}}{\rho_{1}}-\frac{1}{2} \frac{d_{2}^{2}}{\rho_{2}}-\left(\frac{1}{2} C_{1} \rho_{1}^{\prime 2}+D \rho_{1}^{\prime} \rho_{2}^{\prime}+\frac{1}{2} C_{2} \rho_{1}^{\prime 2}\right)=k_{3}$,

where $k_{3}$ is a constant.

Consequently, in one-dimensional system, the profiles of mixture densities are associated with a mechanical system having an equivalent kinetic energy

$$
\frac{1}{2} C_{1} \rho_{1}^{\prime 2}+D \rho_{1}^{\prime} \rho_{2}^{\prime}+\frac{1}{2} C_{2} \rho_{1}^{\prime 2}
$$


and an equivalent potential of forces

$$
-g_{o}\left(\rho_{1}, \rho_{2}\right)-k_{1} \rho_{1}-k_{2} \rho_{2}+\frac{1}{2} \frac{d_{1}^{2}}{\rho_{1}}+\frac{1}{2} \frac{d_{2}^{2}}{\rho_{2}} .
$$

It is possible to use all the tools of analytical dynamics as Lagrange or Hamilton equations, symplectic geometry or Maupertuis methods.

In each bulk, density gradients are null. Eliminating constants $k_{i}, i=\{1,2\}$, dynamical conditions through the interfacial layer yield

$$
\left\{\begin{array}{l}
{\left[g_{, \rho_{1}}\left(\rho_{1}, \rho_{2}\right)\right]=0} \\
{\left[g_{, \rho_{2}}\left(\rho_{1}, \rho_{2}\right)\right]=0} \\
{\left[g-\rho_{1} g_{, \rho_{1}}-\rho_{2} g_{, \rho_{2}}\right]=0}
\end{array}\right.
$$

where $[u]=u_{\beta}-u_{\alpha}$ denotes the difference of values of $u$ between the two mixture bulks $\alpha$ and $\beta$ and

$$
g=g_{o}-\frac{1}{2} \frac{d_{1}^{2}}{\rho_{1}}-\frac{1}{2} \frac{d_{2}^{2}}{\rho_{2}} .
$$

In case of equilibrium at a given temperature $T,\left(d_{1}\right.$ and $d_{2}$ are null), the minimum of the total free energy, with a given total mass for each constituent, yields conditions

$$
\left\{\begin{array}{l}
{\left[\mu_{1}\left(\rho_{1}, \rho_{2}\right)\right]=0} \\
{\left[\mu_{2}\left(\rho_{1}, \rho_{2}\right)\right]=0} \\
{\left[P\left(\rho_{1}, \rho_{2}\right)\right]=0}
\end{array}\right.
$$

where

$$
P\left(\rho_{1}, \rho_{2}\right)=P_{c}+\rho_{1} \mu_{1}\left(\rho_{1}, \rho_{2}\right)+\rho_{2} \mu_{2}\left(\rho_{1}, \rho_{2}\right)-g_{o}\left(\rho_{1}, \rho_{2}\right)
$$

denotes the total thermodynamical pressure of the binary mixture. The constant $P_{c}$, corresponding to the fact that $g_{o}$ is defined to an additive constant, can be defined as the critical pressure term for a value of $g_{o}$ null at the critical point.

Conditions of system (8) (or system (99)) express that the bulks of Gibbs surface $(\Sigma)$ associated with the dynamical volume free energy $g$ (or static volume free energy $g_{o}$ ) correspond to the contact points with a bitangent plane (Fig. $2)$. By adding the term $-\frac{1}{2} \frac{d_{1}^{2}}{\rho_{1}}-\frac{1}{2} \frac{d_{2}^{2}}{\rho_{2}}$ to $g_{o}$, the study of non-dissipative travelling waves of density components of a mixture turns back to an equivalent equilibrium problem. 




Fig. 2. In case of bulk equilibrium, the third coordinate of the point of intersection of the bi-tangent plane with the $g$-axis is $-P\left(\rho_{1}, \rho_{2}\right)$. In dynamical case, the intersection corresponds to the opposite of a dynamical pressure (and defined to an additive constant $P_{c}$ ). Contact points $M_{\alpha}$ and $M_{\beta}$ of the bitangent plane with surface $(\Sigma)$ generate a curve $(\Gamma)$. Along this spinodal curve $(\Gamma)$, the straight line $(D)$ connecting the contact points $M_{\alpha}$ and $M_{\beta}$ associated with the two mixture bulks is the characteristic line of the plane which generates a developable surface. Critical point $C$ corresponds to the collapse of the two contact points at the given temperature $T$.

\section{Integration of the equations of travelling waves}

In Appendix B, we propose an expansion form of the volume energy $g_{o}$ for the binary mixture, near its critical point at temperature $T$, in the form

$$
g_{o}\left(q_{1}, q_{2}\right)=A\left\{\left(B^{2} q_{1}^{2}-q_{2}\right)^{2}+q_{2}^{2}\right\}
$$

where $A$ and $B$ are physical constants, $q_{1}$ and $q_{2}$ are convenient linear combinations of $\rho_{1}-\rho_{1}^{c}$ and $\rho_{2}-\rho_{2}^{c}$ with $\rho_{1}^{c}$ and $\rho_{2}^{c}$ denoting the density values of the two constituents at the critical point (for the sake of simplicity, we denote the volume free energy as $g_{o}$ in all systems of variables). With the change of 
variables,

$$
\mathbf{r}=\left(\begin{array}{c}
r_{1} \\
r_{2}
\end{array}\right) \equiv\left(\begin{array}{c}
\rho_{1}-\rho_{1}^{c} \\
\rho_{2}-\rho_{2}^{c}
\end{array}\right)
$$

and

$$
Y\left(r_{1}, r_{2}\right) \equiv g_{o}\left(r_{1}, r_{2}\right)-\frac{1}{2} \frac{d_{1}^{2}}{r_{1}+\rho_{1}^{c}}-\frac{1}{2} \frac{d_{2}^{2}}{r_{2}+\rho_{2}^{c}}+k_{1}\left(r_{1}+\rho_{1}^{c}\right)+k_{2}\left(r_{2}+\rho_{2}^{c}\right)
$$

system (7) can be written

$$
\mathbf{R} \mathbf{r}^{\prime \prime}=\left(\frac{\partial Y}{\partial \mathbf{r}}\right)^{T}
$$

We denote

$$
\mathbf{P}=\left(\begin{array}{ll}
a & b \\
c & d
\end{array}\right)
$$

where scalars $a, b, c, d$ are depending on the thermo-mechanical properties of the mixture near the point $C$. By the new change of variables,

$$
\mathbf{r}=\mathbf{P} \mathbf{q}, \text { with } \mathbf{q}=\left(\begin{array}{c}
q_{1} \\
q_{2}
\end{array}\right)
$$

and

$$
\begin{aligned}
Y\left(q_{1}, q_{2}\right)= & g_{o}\left(q_{1}, q_{2}\right)-\frac{1}{2} \frac{d_{1}^{2}}{a q_{1}+b q_{2}+\rho_{1}^{c}}-\frac{1}{2} \frac{d_{2}^{2}}{c q_{1}+d q_{2}+\rho_{2}^{c}} \\
& +k_{1}\left(a q_{1}+b q_{2}+\rho_{1}^{c}\right)+k_{2}\left(c q_{1}+d q_{2}+\rho_{2}^{c}\right)
\end{aligned}
$$

the system (11) writes in the form

$$
\widetilde{\mathbf{R}} \mathbf{q}^{\prime \prime}=\left(\frac{\partial Y}{\partial \mathbf{q}}\right)^{T} \quad \text { with } \quad \widetilde{\mathbf{R}}=\left(\begin{array}{cc}
\widetilde{C}_{1} & \widetilde{D} \\
\widetilde{D} & \widetilde{C}_{2}
\end{array}\right)
$$

or

$$
\left\{\begin{array}{l}
\widetilde{C}_{1} q_{1}^{\prime \prime}+\widetilde{D} q_{2}^{\prime \prime}=4 A B^{2}\left(B^{2} q_{1}^{3}-q_{1} q_{2}\right)+\widetilde{k}_{1} \\
\widetilde{D} q_{1}^{\prime \prime}+\widetilde{C_{2}} q_{2}^{\prime \prime}=2 A\left(-B^{2} q_{1}^{2}+2 q_{2}\right)+\widetilde{k}_{2}
\end{array}\right.
$$

where $\widetilde{k}_{1}=a k_{1}+c k_{2}$ and $\widetilde{k}_{2}=b k_{1}+d k_{2}$. Due to the fact $\mathbf{R}$ is symmetric positive definite and $\mathbf{P}$ is not singular, the same holds for $\widetilde{R}=\mathbf{P}^{T} \mathbf{R} \mathbf{P}$. 


\subsection{Case of equilibrium}

\subsubsection{The rescaling process}

The values of constants $\widetilde{k}_{1}$ and $\widetilde{k}_{2}$ must correspond to the singular points of the differential system (17) when $d_{i}=0,(i=1,2)$. These singular points are associated with the two bulks of the binary mixture. Consequently, $\widetilde{k}_{1}=k_{1}=0$ and $\widetilde{k}_{2}=k_{2}=-\tau^{2}$ (see Appendix B, for the meaning of $\tau$ as a "distance" to the critical point).

We consider the following hypothesis $\left(H_{1}\right)$ and $\left(H_{2}\right)$ :

- $\left(H_{1}\right)$ We assume that $q_{1}$ and $q_{2}$ slowly vary as a function of the onedimensional parameter $x$. The hypothesis can be expressed by a change of variable $z=\varepsilon x$, where $\varepsilon$ is an adimensional small parameter $(\varepsilon \ll 1)$. With the following change of variables:

$$
q_{1}(x)=Q_{1}(z) \text { and } q_{2}(x)=Q_{2}(z),
$$

system (13) yields

$$
\left\{\begin{array}{l}
\varepsilon^{2}\left(\widetilde{C}_{1} \ddot{Q}_{1}+\widetilde{D} \ddot{Q}_{2}\right)=4 A B^{2}\left(B^{2} Q_{1}^{3}-Q_{1} Q_{2}\right), \\
\varepsilon^{2}\left(\widetilde{D} \ddot{Q}_{1}+\widetilde{C}_{2} \ddot{Q}_{2}\right)=2 A\left(-B^{2} Q_{1}^{2}+2 Q_{2}\right)-\tau^{2},
\end{array}\right.
$$

where the dot denotes the derivation with respect to $z$. The solution must be a phase transition connecting singular points $M_{\alpha}\left(\tau / B, \tau^{2}\right)$ and $M_{\alpha}\left(-\tau / B, \tau^{2}\right)$.

- $\left(H_{2}\right)$ The density profiles are of small amplitude with respect to the critical densities. Consequently, we look for the solutions in the form

$$
Q_{1}(z)=\varepsilon^{n_{1}} y_{1}(z) \quad \text { and } \quad Q_{2}(z)=\varepsilon^{n_{2}} y_{2}(z)
$$

where $n_{1}$ and $n_{2}$ are to real positive constants. System (14) yields

$$
\left\{\begin{array}{l}
\varepsilon^{2}\left(\widetilde{C}_{1} \varepsilon^{n_{1}} \ddot{y}_{1}+\widetilde{D} \varepsilon^{n_{2}} \ddot{y}_{2}\right)=4 A B^{2}\left(B^{2} \varepsilon^{3 n_{1}} y_{1}^{3}-\varepsilon^{n_{1}+n_{2}} y_{1} y_{2}\right), \\
\varepsilon^{2}\left(\widetilde{D} \varepsilon^{n_{1}} \ddot{y}_{1}+\widetilde{C}_{2} \varepsilon^{n_{2}} \ddot{y}_{2}\right)=2 A\left(-B^{2} \varepsilon^{2 n_{1}} y_{1}^{2}+2 \varepsilon^{n_{2}} y_{2}\right)-\tau^{2} .
\end{array}\right.
$$

The form of the volume energy near the critical point must be conserved by affinity. This assumption corresponds to the universality of the form of the energy near a critical point [10]. Consequently in this rescaling process,

$$
2 n_{1}=n_{2} \quad \text { and } \quad \tau=\varepsilon^{n_{1}} E
$$

where $E$ is a positive constant with physical dimension $M^{1 / 2} L^{-3 / 2}$. System 
(15) yields the equality of same order terms,

$$
\left\{\begin{array}{l}
\varepsilon^{2+n_{1}} \widetilde{C}_{1} \ddot{y}_{1}=\varepsilon^{3 n_{1}} 4 A B^{2}\left(B^{2} y_{1}^{3}-y_{1} y_{2}\right), \\
\varepsilon^{2+n_{1}} \widetilde{D} \ddot{y}_{1}=\varepsilon^{2 n_{1}} 2 A\left(-B^{2} y_{1}^{2}+2 y_{2}-E^{2}\right) .
\end{array}\right.
$$

If we assume $2+n_{1}<2 n_{1}$, Eq. (16) 2 reduces to $\ddot{y}_{1}=0$, which is not physically possible; If we assume $2+n_{1}=2 n_{1}$, Eq. (16) $)_{1}$ reduces to $\ddot{y}_{1}=0$. Then, to obtain non-trivial solutions, we must assume that $2+n_{1}>2 n_{1}$ and $2 A\left(-B^{2} y_{1}^{2}+2 y_{2}-E^{2}\right)=0$; consequently,

$$
y_{2}=\frac{1}{2}\left(B^{2} y_{1}^{2}+E^{2}\right) \text {. }
$$

Now, if we compare $\varepsilon^{2+n_{1}}$ and $\varepsilon^{3 n_{1}}$, we must assume that $n_{1}=1$. System (16) leads to the rescaled system

$$
\left\{\begin{array}{l}
\widetilde{C}_{1} \ddot{y}_{1}=2 A B^{2}\left(B^{2} y_{1}^{3}-E^{2} y_{1}\right) \\
y_{2}=\frac{1}{2}\left(B^{2} y_{1}^{2}+E^{2}\right)
\end{array}\right.
$$

which is equivalent to

$$
\left\{\begin{array}{l}
\frac{\widetilde{C}_{1}}{2} \dot{y}_{1}^{2}=A B^{2}\left(\frac{B^{2}}{2} y_{1}^{4}-E^{2} y_{1}^{2}+k_{4}\right) \\
y_{2}=\frac{1}{2}\left(B^{2} y_{1}^{2}+E^{2}\right),
\end{array}\right.
$$

where $k_{4}$ is a convenient constant of integration.

\subsubsection{Integration of system (17)}

The first equation of system (17) has several possible solutions. In this case we look for phase transition, the solution must be monotonic and must go from an equilibrium point to the other one. The simplest possibility is $k_{4}=E^{4} /\left(2 B^{2}\right)$. The integration of Eq. (17) 1 yields

$$
y_{1}(z)= \pm \frac{E}{B} \tanh \left(E \sqrt{\frac{2 A B^{2}}{\widetilde{C}_{1}} x}\right) .
$$

Consequently, with the change of orientation of the $x$-axis, we get

$$
\left\{\begin{array}{l}
q_{1}(x)=\frac{\tau}{B} \tanh \left(\tau \sqrt{\left.\frac{2 A B^{2}}{\widetilde{C}_{1}} x\right)}\right. \\
q_{2}(x)=\frac{\tau^{2}}{2}\left\{1+\tanh ^{2}\left(\tau \sqrt{\frac{2 A B^{2}}{\widetilde{C}_{1}} x}\right)\right\} .
\end{array}\right.
$$


Due to the changes of variables (10), (12), we obtain the general form of the phase transitions

$$
\left\{\begin{array}{l}
\rho_{1}(x)=\rho_{1}^{c}+a_{1} \tanh \left(\frac{x}{L}\right)+b_{1}\left\{1+\tanh ^{2}\left(\frac{x}{L}\right)\right\} \\
\rho_{2}(x)=\rho_{2}^{c}+a_{2} \tanh \left(\frac{x}{L}\right)+b_{2}\left\{1+\tanh ^{2}\left(\frac{x}{L}\right)\right\} .
\end{array}\right.
$$

Depending on the two cases of boundary conditions, we obtain

$1^{\text {st }}$ Case: When $\lim _{x \rightarrow-\infty} \rho_{i}(x)=\rho_{i}^{\alpha}$ and $\lim _{x \rightarrow+\infty} \rho_{i}(x)=\rho_{i}^{\beta} \quad$ with $i=1,2$, then

$$
a_{i}=\frac{1}{2}\left(\rho_{i}^{\beta}-\rho_{i}^{\alpha}\right), \quad b_{i}=\frac{1}{4}\left(\rho_{i}^{\alpha}-2 \rho_{i}^{c}+\rho_{i}^{\beta}\right) .
$$

$2^{n d}$ Case: When $\lim _{x \rightarrow-\infty} \rho_{i}(x)=\rho_{i}^{\beta}$ and $\lim _{x \rightarrow+\infty} \rho_{i}(x)=\rho_{i}^{\alpha}$ with $i=1,2$, then

$$
a_{i}=\frac{1}{2}\left(\rho_{i}^{\alpha}-\rho_{i}^{\beta}\right), \quad b_{i}=\frac{1}{4}\left(\rho_{i}^{\alpha}-2 \rho_{i}^{c}+\rho_{i}^{\beta}\right) .
$$

Let us note that the constants $a_{i}$ and $b_{i}$ depend on the thermodynamical behavior of the mixture and they may be positive or negative.

\subsubsection{The different cases}

The variation of $\rho_{1}(x)$ and $\rho_{2}(x)$ in system (18) yields the different cases. They are summarized on Fig. 3 as domains delimited by the bisectrices of axes $a_{i}$ and $b_{i}$ and we represent the different possible variations of each component on Fig. 4. We see, as it is known by other methods in physical chemistry that the density profiles in interfacial densities are not necessary homogeneous for mixtures of fluids [37,38].

\subsection{Case of travelling waves}

In the case of motion, we check with travelling waves submitted to viscosity; in this case, system (5) can be modified to take account of the viscosity in each component of the mixture

$$
\left\{\begin{array}{l}
\mathbf{a}_{1}=\operatorname{grad}\left\{C_{1} \Delta \rho_{1}+D \Delta \rho_{2}-g_{o, \rho_{1}}\right\}+\frac{1}{\rho_{1}} \operatorname{div} \sigma_{1} \\
\mathbf{a}_{2}=\operatorname{grad}\left\{D \Delta \rho_{1}+C_{2} \Delta \rho_{2}-g_{o, \rho_{2}}\right\}+\frac{1}{\rho_{2}} \operatorname{div} \sigma_{2},
\end{array}\right.
$$




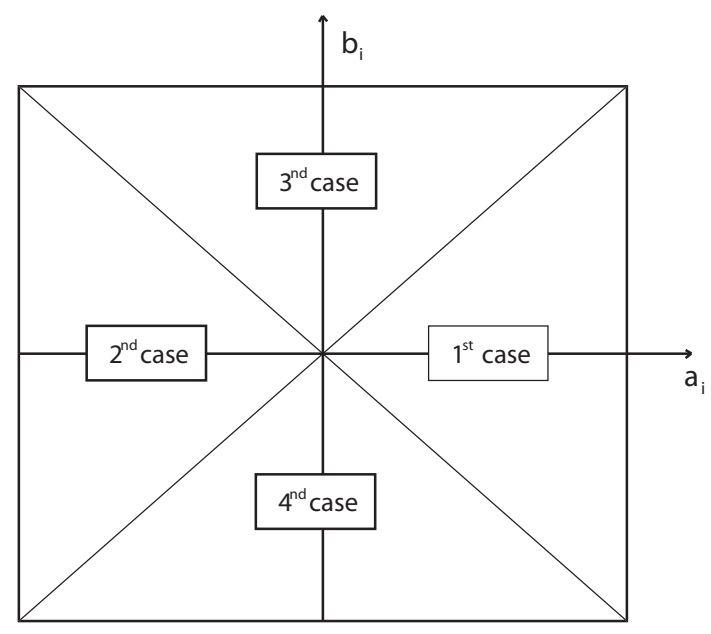

Fig. 3. The different possible cases for each mixture component are presented on the graph associated with the $a_{i}$-axis and $b_{i}$-axis. Points on axis $a_{i}$ where $\rho_{i}^{\alpha}=\rho_{i}^{\beta}$ are forbidden; this will not be the case in dynamics.
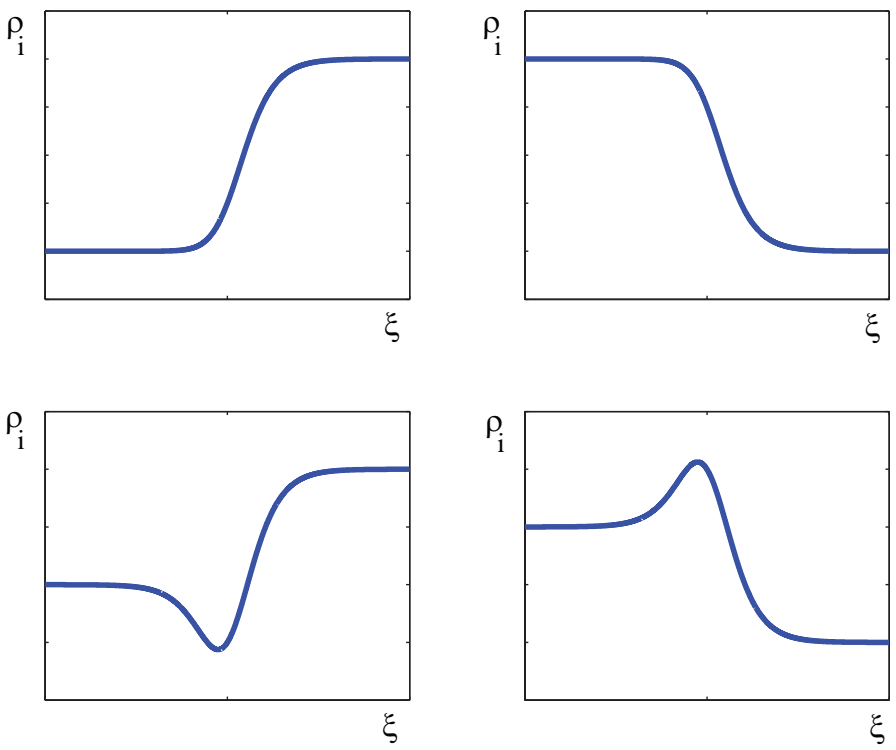

Fig. 4. Sketch of the different possible forms of phase transition for each mixture component are presented on the graph with densities $\rho_{i}$ versus $\xi$.

where $\sigma_{i}\{i=1,2\}$ is the viscous stress tensor associated with component $i$. Consequently we obtain in variables $q_{1}$ and $q_{2}$, the system of equations of motions in one-dimensional system with $\sigma_{i}=\nu_{i} v_{i}^{\prime \prime}$, where $\nu_{i}>0$ is the coefficient of viscosity of the component $i$ [39]. The system can be written by using one dimensional derivation in $\operatorname{div} \sigma_{i}$, by integration as in section 4.1 and with the 
fact that $\rho_{i} \approx \rho_{i}^{c}$,

$$
\left\{\begin{array}{l}
\widetilde{C}_{1} q_{1}^{\prime \prime}+\widetilde{D} q_{2}^{\prime \prime}=4 A B^{2}\left(B^{2} q_{1}^{3}-q_{1} q_{2}\right) \\
+\frac{1}{2} \frac{a d_{1}^{2}}{\left(\rho_{1}^{c}\right)^{2}}+\frac{1}{2} \frac{c d_{2}^{2}}{\left(\rho_{2}^{c}\right)^{2}}-\frac{a d_{1} \nu_{1}}{\left(\rho_{1}^{c}\right)^{3}} q_{1}^{\prime}-\frac{c d_{2} \nu_{2}}{\left(\rho_{1}^{c}\right)^{3}} q_{2}^{\prime}+\widetilde{k}_{1} \\
\widetilde{D} q_{1}^{\prime \prime}+\widetilde{C_{2}} q_{2}^{\prime \prime}=2 A\left(-B^{2} q_{1}^{2}+2 q_{2}\right) \\
+\frac{1}{2} \frac{b d_{1}^{2}}{\left(\rho_{1}^{c}\right)^{2}}+\frac{1}{2} \frac{d d_{2}^{2}}{\left(\rho_{2}^{c}\right)^{2}}-\frac{b d_{1} \nu_{1}}{\left(\rho_{1}^{c}\right)^{3}} q_{1}^{\prime}-\frac{d d_{2} \nu_{2}}{\left(\rho_{1}^{c}\right)^{3}} q_{2}^{\prime}+\widetilde{k}_{2} .
\end{array}\right.
$$

\subsubsection{The rescaling process}

We can make an analogous rescaling process as in Section 4.1.1. The difference only comes from scalars $d_{1}$ and $d_{2}$. They must be of small amplitude; consequently, we look for constants in the form

$$
D_{1}=\varepsilon^{n_{3}} d_{1} \quad \text { and } \quad D_{2}=\varepsilon^{n_{4}} d_{2}
$$

where constants $n_{3}$ and $n_{4}$ are positive. To obtain convenient solutions we must have

$$
d_{i}=D_{i} \varepsilon^{\frac{3 n_{1}}{2}},(i=1,2), \widetilde{k}_{1}=\varepsilon^{3 n_{1}} \widetilde{K}_{1}, \widetilde{k}_{2}=\varepsilon^{2 n_{1}} \widetilde{K}_{2}, n_{1}=1
$$

Then, the terms $\frac{d_{1} \nu_{1}}{\left(\rho_{1}^{c}\right)^{3}} q_{1}^{\prime}=\frac{D_{1} \nu_{1}}{\left(\rho_{1}^{c}\right)^{3}} \varepsilon^{\left(3+\frac{1}{2}\right)} \dot{y}_{1}$ and $\frac{d_{2} \nu_{2}}{\left(\rho_{2}^{c}\right)^{3}} q_{2}^{\prime}=\frac{D_{2} \nu_{2}}{\left(\rho_{2}^{c}\right)^{3}} \varepsilon^{\left(4+\frac{1}{2}\right)} \dot{y}_{2}$ which are of order greater than the other terms in system (19), are negligible. Finally, system (19) yields

$$
\left\{\begin{array}{l}
\widetilde{C}_{1} \dot{y}_{1}^{2}=A B^{2}\left(B^{2} y_{1}^{4}+\frac{\widetilde{K}_{2}}{A} y_{1}^{2}+\widetilde{K}_{3} y_{1}+\widetilde{K}_{4}\right), \\
y_{2}=\frac{1}{2}\left(B^{2} y_{1}^{2}-\frac{\widetilde{K}_{2}}{2 A}\right),
\end{array}\right.
$$

where $\widetilde{K}_{3}=\frac{2}{A B^{2}}\left(\frac{a D_{1}^{2}}{2\left(\rho_{1}^{c}\right)^{2}}+\frac{c D_{2}^{2}}{2\left(\rho_{2}^{c}\right)^{2}}+\widetilde{K}_{1}\right)$ and $\widetilde{K}_{4}$ is a new constant of integration.

\subsubsection{Integration of system (20)}

A qualitative study of Eq. $(20){ }_{1}$ can be considered. Constant $A$ is strictly positive. The discussion comes from

$$
\dot{y}_{1}^{2}=f\left(y_{1}\right)-g\left(y_{1}\right)
$$


where

$$
f\left(y_{1}\right)=\frac{A B^{4}}{\widetilde{C}_{1}}\left(y_{1}^{2}+\frac{\widetilde{K}_{2}}{2 A B^{2}}\right)^{2}
$$

represents a quartic and

$$
g\left(y_{1}\right)=\frac{A B^{2}}{\widetilde{C}_{1}}\left(-\widetilde{K}_{3} y_{1}-\widetilde{K}_{4}+\frac{\widetilde{K}_{2}^{2}}{4 A^{2} B^{2}}\right)
$$

represents a straight line. If $\widetilde{K}_{2} \geq 0, f\left(y_{1}\right)$ is a convex quartic and only periodic solutions are possible. Consequently $\widetilde{K}_{2}<0$.

Two interesting cases can be considered:

a) The straight line (22) is bitangent to the quartic (21). The solution of Eq. $(20)_{1}$ is a travelling wave of phase transition in the same form than the two first curves of Fig. 4. We must have

$$
\widetilde{K}_{3}=0, \quad \widetilde{K}_{4}=\frac{\widetilde{K}_{2}^{2}}{4 A^{2} B^{2}} \quad \text { and consequently } \quad \widetilde{K}_{1}=-\frac{a D_{1}^{2}}{2\left(\rho_{1}^{c}\right)^{2}}-\frac{c D_{2}^{2}}{2\left(\rho_{2}^{c}\right)^{2}}
$$

Then,

$$
\left\{\begin{array}{l}
q_{1}(\xi)=\frac{\varkappa}{B} \tanh \left(\varkappa \sqrt{\left.\frac{2 A B^{2}}{\widetilde{C}_{1}} \xi\right)}\right. \\
q_{2}(\xi)=\frac{\varkappa^{2}}{2}\left\{1+\tanh ^{2}\left(\varkappa \sqrt{\frac{2 A B^{2}}{\widetilde{C}_{1}}} \xi\right)\right\} .
\end{array}\right.
$$

We note that $\varkappa$ plays the role of $\tau$ in the static case. The solutions in variables $\rho_{1}$ and $\rho_{2}$ are in the same form than in Section 4.1.2 and the same sort of density profiles can be observed

$$
\left\{\begin{array}{l}
\rho_{1}(\xi)=\rho_{1}^{c}+\frac{a \varkappa}{B} \tanh \left(\frac{\xi}{L_{1}}\right)+\frac{b \varkappa^{2}}{2}\left\{1+\tanh ^{2}\left(\frac{\xi}{L_{1}}\right)\right\} \\
\rho_{2}(\xi)=\rho_{2}^{c}+\frac{c \varkappa}{B} \tanh \left(\frac{\xi}{L_{1}}\right)+\frac{d \varkappa^{2}}{2}\left\{1+\tanh ^{2}\left(\frac{\xi}{L_{1}}\right)\right\} .
\end{array}\right.
$$

We have seen that to obtain travelling waves near a critical point, the viscosity terms have to be negligible with respect to the others. This result is in accordance with dispersive waves as the ones obtained by using the Korteweg-de Vries equation [43]. 


\section{Conclusion}

The equations of binary mixtures of fluids are considered in the one-dimensional case. By using an asymptotic expansion of the volume free energy near a critical point and by taking the density gradients into account, we get, in continuum mechanics, a dynamical system representing the equations of travelling waves for the two-fluid components. A rescaling process associated with the universality of the energy form near a critical point allows to obtain different sorts of travelling waves of density as well as in conservative and dissipative cases. We can discuss different possible phase transitions according to the values of phases densities of components and the characteristic of the mixture.

Now, we consider some physical comments. For given densities, at time $t=0$, the velocities of the mixture components are deduced from the mass balance equations (6). Then, an arbitrary constant $s$ is introduced in the velocity fields:

$$
v_{i}(x)=\frac{d_{i}}{\rho_{i}(x)}+s
$$

and at any time $t$ the relation leads to

$$
v_{i}(x-s t)=\frac{d_{i}}{\rho_{i}(x-s t)}+s .
$$

The initial conditions yield this arbitrary velocity $s$.

First case: interface propagation - By crossing the interface (interface means the domain where the densities drastically change), the volume changes. This phenomenon cannot occur in a closed tube with constant volume, but only in a tube with one closed end. It is similar to a phenomenon of vaporization or condensation.

One imagines that the other side of the tube is closed with a piston whose displacement may be imposed. The component velocities at the fixed end are zero in bulk $\beta$; this determines the constant $s$ thanks to the condition

$$
s=\frac{d_{1}}{\rho_{1}^{\beta}}=\frac{d_{2}}{\rho_{2}^{\beta}} .
$$

Due to the fact that $\rho_{1}^{\beta} \simeq \rho_{1}^{c}$ and $\rho_{2}^{\beta} \simeq \rho_{2}^{c}$, we get

$$
s=\frac{d_{1}}{\rho_{1}^{c}}=\frac{d_{2}}{\rho_{2}^{c}}
$$

and the values of $d_{1}$ and $d_{2}$ are not independent.

Second case: solitary waves - The volume of the interface only moves in one bulk phase and remains constant. Such a wave may move in a closed tube as 
Natterer's one [6]. At the ends which are assumed far from the interface, the velocities in the bulk phase are zero and we still obtain Eq. (23) such that the values of $d_{1}$ and $d_{2}$ are not independent.

\section{Appendix A: Proof of system (5)}

Hamilton's principle - variational form of the principle of virtual powers allows us to derive the equations of motions. The variations of motions of particles are deduced from the functions

$$
\mathbf{X}_{i}=\Psi_{i}\left(\mathbf{z} ; \beta_{i}\right), \quad i \in\{1,2\}
$$

for which $\beta_{i}$ are two parameters defined in a neighborhood of zero; they are associated with two families of virtual motions of the mixture. The real motions correspond to $\beta_{i}=0: \quad \Psi_{i}(\mathbf{z} ; 0)=\Phi_{i}(\mathbf{z})$.

Virtual material displacements associated with any variation of real motions can be written as [16]

$$
\delta \mathbf{X}_{i}=\left.\frac{\partial \Psi_{i}}{\partial \beta_{i}}\right|_{\beta_{i}=0}
$$

Such a variation is dual with Serrin's [40. This has been studied in [16] and corresponds to the natural variation of the motion in a Lagrangian representation.

Let us denote $\xi_{i}=\delta_{i} \mathbf{X}_{i}$; we deduce the two relations in Lagrangian variables [8, 30, 40]

$$
\delta_{i} \mathbf{v}_{i}=-\mathbf{F}_{i} \frac{\partial \xi_{i}}{\partial t}, \quad \delta_{i} \rho_{i}=\rho_{i} \operatorname{div}_{o i} \xi_{i}+\frac{\rho_{i}}{\rho_{o i}} \frac{\partial \rho_{o i}}{\partial \mathbf{X}_{i}} \xi_{i}
$$

where $\partial / \partial \mathbf{X}_{i}$ is the linear form associated with the gradient and $\operatorname{div}_{o i}$ is the divergence operator on $\mathcal{D}_{o i}$. Assuming that terms on the edge of $\mathcal{W}$ are zero, we obtain

$\delta_{i} a=\int_{t_{1}}^{t_{2}} \int_{\mathcal{D}_{t}}\left\{\left(\frac{1}{2} \mathbf{v}_{i}^{2}-e_{, \rho_{i}}-\Omega_{i}\right) \delta_{i} \rho_{i}+\rho_{i} \mathbf{v}_{i}^{T} \delta_{i} \mathbf{v}_{i}-\left\{\frac{\partial}{\partial x_{\gamma}}\left(\frac{\partial e}{\partial \rho_{i, \gamma}}\right)\right\} \delta_{i} \rho_{i}\right\} d v d t$

or

$$
\delta_{i} a=\int_{t_{1}}^{t_{2}} \int_{\mathcal{D}_{o i}} \rho_{o i}\left\{R_{i} \operatorname{div}_{o i} \xi_{i}+\frac{R_{i}}{\rho_{o i}} \frac{\partial \rho_{o i}}{\partial \mathbf{X}_{i}} \xi_{i}-\mathbf{v}_{i}^{T} \mathbf{F}_{i} \frac{\partial \xi_{i}}{\partial t}\right\} d v_{o i} d t
$$

where $R_{i}=\frac{1}{2} \mathbf{v}_{i}^{2}-\mu_{i}-\Omega_{i}$. But,

$$
\operatorname{div}_{o i}\left(\rho_{o i} R_{i} \xi_{i}\right)=\rho_{o i} R_{i} \operatorname{div}_{o i} \xi_{i}+R_{i} \frac{\partial \rho_{o i}}{\partial \mathbf{X}_{i}} \xi_{i}+\rho_{o i} \frac{\partial R_{i}}{\partial \mathbf{X}_{i}} \xi_{i}
$$


So, the terms given by integration on the edge of $\mathcal{D}_{\text {oi }}$ being zero,

$$
\delta_{i} a=\int_{t_{1}}^{t_{2}} \int_{\mathcal{D}_{o i}} \rho_{o i}\left\{-\frac{\partial R_{i}}{\partial \mathbf{X}_{i}}+\frac{\partial}{\partial t}\left(\mathbf{v}_{i}^{T} \mathbf{F}_{i}\right)\right\} \xi_{i} d v_{o i} d t .
$$

Finally, for each constituent,

$$
\frac{\partial}{\partial t}\left(\mathbf{v}_{i}^{T} \mathbf{F}_{i}\right)=\frac{\partial R_{i}}{\partial \mathbf{X}_{i}}
$$

and by using the relation

$$
\frac{\partial}{\partial t}\left(\mathbf{v}_{i}^{T} \mathbf{F}_{i}\right)=\mathbf{a}_{i}^{T} \mathbf{F}_{i}+\mathbf{v}_{i}^{T} \frac{\partial \mathbf{v}_{i}}{\partial \mathbf{X}_{i}}
$$

we obtain

$$
\mathbf{a}_{i}^{T} \mathbf{F}_{i}+\frac{1}{2} \frac{\partial \mathbf{v}_{i}^{2}}{\partial \mathbf{X}_{i}}=\frac{\partial R_{i}}{\partial \mathbf{X}_{i}} \quad \text { or } \quad \mathbf{a}_{i}^{T}+\frac{\partial}{\partial \mathbf{x}}\left(\mu_{i}+\Omega_{i}\right)=0 .
$$

This leads to the vectorial form (4)

$$
\mathbf{a}_{i}+\operatorname{grad}\left(\mu_{i}+\Omega_{i}\right)=0, i=\{1,2\}
$$

\section{Appendix B: The volume free energy of a binary mixture near critical conditions}

In this Appendix, thanks to a direct method of differential geometry, we obtain the form of the free energy of a binary mixture near a critical point. In physical chemistry, the form is presented thanks to the knowledge of the thermodynamic coordinates of the bulks when we are near a critical point [37]. In this new presentation, we take only account of conditions of equilibrium of bulks when the critical point marks the limit of their coexistence. Such a method implies general physical consequences as in one component fluid when the two variables are densities of matter and entropy but might also be extended in other topics when different equilibrium states collapse only in one state as in finance or biology.

The volume free energy of binary mixture at temperature $T$ is taken in the form:

$$
g_{o}=g_{o}\left(\rho_{1}, \rho_{2}\right),
$$

where $\rho_{1}, \rho_{2}$ are the densities of the two components. The following relations between the chemical potentials $\mu_{1}, \mu_{2}$ of the two constituents and $g_{o}$ hold

$$
\mu_{1}=\left(g_{o}\right)_{\rho_{1}}^{\prime}, \quad \mu_{2}=\left(g_{o}\right)_{\rho_{2}}^{\prime}
$$


and the thermodynamical pressure is given by

$$
P=-g_{o}+\rho_{1} \mu_{1}+\rho_{2} \mu_{2}+P_{c}=-g_{o}+\rho_{1}\left(g_{o}\right)_{\rho_{1}}^{\prime}+\rho_{2}\left(g_{o}\right)_{\rho_{2}}^{\prime}+P_{c} .
$$

As the function $g_{o}$ is twice differentiable, the geometrical representation of $g_{o}$ by a surface $\Sigma$ admits a tangent plane at any point. The normal vector to the plane has the direction coefficients $\left(-\mu_{1}-\mu_{2}, 1\right)$.

The phase equilibrium of two bulks $\alpha, \beta$ is described by the conditions

$$
T(\alpha)=T(\beta), \mu_{1}(\alpha)=\mu_{1}(\beta), \mu_{2}(\alpha)=\mu_{2}(\beta), P(\alpha)=P(\beta) .
$$

It is noticeable that, in this case, the third coordinate of the intersection point of the bitangent plane with the third axis is $-P\left(\rho_{1}, \rho_{2}, T\right)+P_{c}$ (see Fig. 2). The contact points $M_{\alpha}, M_{\beta}$ of the bitangent plane to $\Sigma$ generate two curves $\left(\Gamma_{\alpha}\right),\left(\Gamma_{\beta}\right)$ respectively. The curve $(\Gamma)=\left(\Gamma_{\alpha}\right) \cup\left(\Gamma_{\beta}\right)$ is the spinodal curve of the phase transition. The case with only one contact point corresponds to a mixture with only one bulk while two contact points with a bitangent plane correspond to a two-bulk equilibrium mixture.

When, at a given temperature $T, M_{\alpha}$ tends to $M_{\beta}$, the limit point $C$ is a critical point corresponding to the fact that two bulks collapse into one bulk. The study of the mixture equilibrium near the critical point $C$, at a given temperature $T$, is closely related to the shape of surface $\Sigma$ in the vicinity of $C$. So it is interesting to prove some theorems giving us information about the geometry of the surface $\Sigma$ in the vicinity of $C$.

Theorem. The tangent plane to the surface $\Sigma$ at point $C$ is the osculatory plane of $(\Gamma)$.

Proof

Let $\overrightarrow{O M}=\overrightarrow{\mathbf{F}(\tau)}$ the equation of curve $(\Gamma)$ so that $\overrightarrow{\mathbf{F}(0)}=\overrightarrow{O C}$. The tangent plane at $M_{\alpha}$ or $M_{\beta}$ is

$$
\overrightarrow{M_{\alpha} M_{\beta}}=\overrightarrow{\mathbf{F}\left(\tau_{\beta}\right)}-\overrightarrow{\mathbf{F}(0)}-\overrightarrow{\mathbf{F}\left(\tau_{\alpha}\right)}+\overrightarrow{\mathbf{F}(0)}
$$

or, in the $(\Gamma)$ representation,

$$
\overrightarrow{M_{\alpha} M_{\beta}}=\left(\tau_{\beta}-\tau_{\alpha}\right) \overrightarrow{\mathbf{F}^{\prime}(0)}+\frac{1}{2}\left(\tau_{\beta}^{2}-\tau_{\alpha}^{2}\right) \overrightarrow{\mathbf{F}^{\prime \prime}(0)}+\ldots
$$

and

$$
\overrightarrow{\mathbf{F}^{\prime}\left(\tau_{\alpha}\right)}=\overrightarrow{\mathbf{F}^{\prime}(0)}+\tau_{\alpha} \overrightarrow{\mathbf{F}^{\prime \prime}(0)}+\ldots
$$

It follows

$$
\overrightarrow{\mathbf{F}^{\prime}\left(\tau_{\alpha}\right)} \wedge \overrightarrow{M_{\alpha} M_{\beta}}=\frac{1}{2}\left(\tau_{\alpha}-\tau_{\beta}\right)^{2} \overrightarrow{\mathbf{F}^{\prime}(0)} \wedge \overrightarrow{\mathbf{F}^{\prime \prime}(0)}+\ldots
$$


When $\tau_{\alpha}$ and $\tau_{\beta}$ tends to 0 , the plane $\left(M_{\alpha}, \overrightarrow{M_{\alpha} M_{\beta}}, \overrightarrow{\mathbf{F}^{\prime}\left(\tau_{\alpha}\right)}\right)$ tends to the osculatory plane to $(\Gamma)$ at $C$ because the vector $\frac{2 \overrightarrow{\mathbf{F}^{\prime}\left(\tau_{\alpha}\right)} \wedge \overrightarrow{M_{\alpha} M_{\beta}}}{\left(\tau_{\alpha}-\tau_{\beta}\right)^{2}}$ admits the limit $\overrightarrow{\mathbf{F}^{\prime}(0)} \wedge \overrightarrow{\mathbf{F}^{\prime \prime}(0)}$.

Theorem. The parametric representation of curve $\Gamma$ in Frenet frame at $C$ is

$$
x=\tau, y=\tau^{2}, z=\tau^{4} ; \quad \tau \in v(0) .
$$

Proof.

Let $C$ an ordinary point of $(\Gamma)$. In a neighborhood of $C$, we have

$$
x=\tau, y=\tau^{2}, z=\tau^{3} ; \quad \tau \in v(0)
$$

such that $\tau=0$ corresponds to $C$. Let $M_{\alpha}$ and $M_{\beta}$ two points of $\Gamma$ with $\tau_{\alpha} \tau_{\beta}<0$. Eqs. (24) lead to

$$
\left(\overrightarrow{\mathbf{F}^{\prime}\left(\tau_{\alpha}\right)} \wedge \overrightarrow{M_{\alpha} M_{\beta}}\right)=\left(\begin{array}{c}
-\tau_{\alpha}^{3}+2 \tau_{\alpha} \tau_{\beta}^{2}-\tau_{\alpha}^{2} \tau_{\beta} \\
2 \tau_{\alpha}^{2}-\tau_{\beta}^{2}-\tau_{\alpha} \tau_{\beta} \\
\tau_{\beta}-\tau_{\alpha}
\end{array}\right)
$$

and by changing $\tau_{\beta}$ with $\tau_{\alpha}$,

$$
\left(\overrightarrow{\mathbf{F}^{\prime}\left(\tau_{\beta}\right)} \wedge \overrightarrow{M_{\beta} M_{\alpha}}\right)=\left(\begin{array}{c}
-\tau_{\beta}^{3}+2 \tau_{\beta} \tau_{\alpha}^{2}-\tau_{\alpha} \tau_{\beta}^{2} \\
2 \tau_{\beta}^{2}-\tau_{\alpha}^{2}-\tau_{\beta} \tau_{\alpha} \\
\tau_{\alpha}-\tau_{\beta}
\end{array}\right)
$$

The two vectors in Eqs.(25, 26) are parallel if and only if

$$
\left\{\begin{array}{l}
\tau_{\alpha}^{2}+\tau_{\beta}^{2}-2 \tau_{\alpha} \tau_{\beta}=0 \\
\tau_{\alpha}^{3}+\tau_{\beta}^{3}-\tau_{\alpha} \tau_{\beta}^{2}-\tau_{\beta} \tau_{\alpha}^{2}=0
\end{array}\right.
$$

Equation (27) $)_{1}$ yields $\tau_{\alpha}=\tau_{\beta}$ and then $(27)_{2}$ is verified. This means that any relation $\tau_{\beta}=\phi\left(\tau_{\alpha}\right)$ different from identity is not allowed and $\tau_{\alpha} \tau_{\beta}<0$ is not possible. Then, the critical point $C$ cannot be an ordinary point of $(\Gamma)$. Let us now consider the simplest singular case for $\Gamma$ at $C$

$$
x=\tau, y=\tau^{2}, z=\tau^{4} .
$$

The parallelism conditions for vectors $\overrightarrow{\mathbf{F}^{\prime}\left(\tau_{\alpha}\right)} \wedge \overrightarrow{M_{\alpha} M_{\beta}}$ and $\overrightarrow{\mathbf{F}^{\prime}\left(\tau_{\beta}\right)} \wedge \overrightarrow{M_{\beta} M_{\alpha}}$ are 
equivalent to

$$
\left\{\begin{array}{l}
-2 \tau_{\alpha}^{4}-2 \tau_{\alpha}^{3} \tau_{\beta}+2 \tau_{\alpha}^{2} \tau_{\beta}^{2}+2 \tau_{\alpha} \tau_{\beta}^{3}=2 \tau_{\beta}^{4}+2 \tau_{\beta}^{3} \tau_{\alpha}-2 \tau_{\beta}^{2} \tau_{\alpha}^{2}-2 \tau_{\beta} \tau_{\alpha}^{3} \\
3 \tau_{\alpha}^{3}-\tau_{\alpha}^{2} \tau_{\beta}-\tau_{\alpha} \tau_{\beta}^{2}-\tau_{\beta}^{3}=-3 \tau_{\beta}^{3}+\tau_{\beta}^{2} \tau_{\alpha}+\tau_{\beta} \tau_{\alpha}^{2}+\tau_{\alpha}^{3}
\end{array}\right.
$$

Equation (28) $)_{2}$ implies $2\left(\tau_{\alpha}+\tau_{\beta}\right)\left(\tau_{\alpha}-\tau_{\beta}\right)^{2}=0$ which has, for $\tau_{\alpha} \neq \tau_{\beta}$, the unique possible solution $\tau_{\alpha}=-\tau_{\beta}$ and then $(28)_{1}$ is satisfied.

The equations of the projection $\left(\Gamma^{\prime}\right)$ of the curve $(\Gamma)$ on the plane $y=\tau_{\alpha}^{2}$ are

$$
x=\tau, \quad z=\tau^{4}
$$

and the straight line $M_{\alpha} M_{\beta}$ remains bitangent to $\left(\Gamma^{\prime}\right)$.

Consequently, in the plane $y=\tau_{\alpha}^{2}$, the main part of the equation of $\left(\Gamma^{\prime}\right)$ is written in the form

$$
z=H\left(\tau_{\alpha}\right)\left(x-\tau_{\alpha}\right)^{2}\left(x+\tau_{\alpha}\right)^{2}+\tau_{\alpha}^{4} \quad \text { with } \quad H(0) \neq 0
$$

In adimensional form, $H(0)=1$ and the main part of the equation of surface $\Sigma$ generated by this curve in a neighborhood of the critical point $C$, when point $C$ is taken as origin of the axes and $C x y$ is the osculatory plane to $\Sigma$ at $C$, is

$$
z=\left(x^{2}-y\right)^{2}+y^{2}
$$

The dimensions of $x$ and $y$ are different. In an adimensional system of axes, the equation writes

$$
z=A\left\{\left(B^{2} x^{2}-y\right)^{2}+y^{2}\right\}
$$

\section{References}

[1] A. Bedford, D.S. Drumheller, Recent advances. Theories of immiscible and structured mixtures, Int. J. Engng. Sci., 21 (1983) 863-960.

[2] V.L. Berdichevsky, Construction of models of continuous media by means of the variational principle, J. Appl. Math. Mech. 30 (1966) 510-530.

[3] G. Boillat, Non linear hyperbolic fields and waves in Recent mathematical methods in nonlinear wave propagation, Ruggeri T. (Ed.), Lecture Notes in Mathematics 1640, Springer, Berlin, 1996.

[4] V. Bongiorno, L.E. Scriven, H.T. Davis, Molecular theory of fluid interfaces, J. Coll. Int. Sci. 57 (1976) 462-475.

[5] R.M. Bowen, Theory of mixtures in Continuum physics III, Ed. A.C. Eringen, Academic Press, London, 1976. 
[6] Bruhat G., Cours de Physique Générale, Thermodynamique, Masson, Paris, 1968.

[7] J.W. Cahn, J.E. Hilliard, Free energy of a non-uniform system, J. Chemical Physics, 31 (1959) 688-699.

[8] P. Casal, H. Gouin , Non-isothermal liquid-vapour interfaces, J. Th. Appl. Mech. (Journal de mécanique théorique et appliquée), 7 (1988) 689-718.

[9] S.R. de Groot, P. Mazur, Non-Equilibrium Thermodynamics, Wiley Interscience, London, 1962.

[10] C. Domb, The critical point, Taylor and Francis, London, 1996.

[11] J.E. Dunn, J. Serrin, On the thermomechanics of interstitial working, Arch. Rat. Mech. Anal., 88 (1985) 95-133.

[12] M.E. Eglit, A generalization of the model of an ideal compressible fluid, J. Appl. Math. Mech., 29 (1965) 351-354.

[13] G. Emschwiller, Chimie-Physique, P.U.F., Paris, 1964.

[14] P. Galdi, D.D. Joseph, L. Preziosi, S. Rionero, Mathematical problems for miscible incompressible fluids with Korteweg stresses, Eur. J. Mech., B/Fluids, 10 (1991) 253-267.

[15] P. Germain, La méthode des puissances virtuelles en mécanique des milieux continus, Journal de mécanique, 12 (1973) 235-275.

[16] H. Gouin, Variational theory of mixtures in continuum mechanics, Eur. J. Mech., B/Fluids, 9 (1990) 469-491, http://arXiv:0807.4519.

[17] H. Gouin, J.M. Delhaye, Material waves of a fluid in the vicinity of the critical point in Symposium on waves in liquid/gas and liquid/vapor two-phase systems, S. Morioka S., Wijngaarden L. (Eds.), Kluwer Publ., Netherlands, 1995.

[18] H. Gouin, Energy of interaction between solid surfaces and liquids, J. Phys. Chem. B 102 (1998) 1212-1218, http://arXiv:0801.4481.

[19] H. Gouin, T. Ruggeri, Mixtures of fluids involving entropy gradients and acceleration waves in interfacial layers, Eur. J. Mech., B/Fluids, 24 (2005) 596613, http://arXiv:0801.2096.

[20] J. Hadamard, Leçons sur la propagation des ondes et les équations de l'hydrodynamique, Chelsea Publ., New York 1949.

[21] R. Hagan, J. Serrin, Dynamics Changes of Phase in a van der Waals Fluid, In New Perspectives in Thermodynamics, Editor: J. Serrin, Publications of the IMA, (1986), 241-260.

[22] J.W. Herivel, The derivation of the equations of motion of an ideal fluid by Hamilton's principle, Proc. Camb. Philos. Soc. 51 (1955) 344-349.

[23] P.C. Hohenberg, B.I. Halperin, Theory of Dynamic Critical Phenomena, Rev. Modern Physics, 49 (1977) 435-501. 
[24] J. Israelachvili, Intermolecular Forces, Academic Press, New York, 1992.

[25] D.D. Joseph, Fluid Dynamics of two miscible liquids with slow diffusion and Korteweg stresses. Eur. J. Mech., B/Fluids, 9 (1990) 565-596.

[26] P.H. van Konynenburg, R.L. Scott, Critical Lines and Phase Equilibria in binary van der Walls mixtures, Phil. Trans. R. Soc. Lond. A 298 (1980) 495-540.

[27] J. Korteweg, Sur la forme que prennent les équations du mouvement des fluides si l'on tient compte des forces capillaires, Archives Néerlandaises 2, $\mathrm{n}^{\circ} 6$ (1901) $1-24$.

[28] L.D. Landau, E. Lifshits, Fluid Mechanics, Pergamon Press, London, 1989.

[29] V. Levitch, Physicochemical Hydrodynamics, Prentice-Hall, Englewood Cliffs, New Jersey, 1962.

[30] C.C. Lin, A new variational principle for isoenergetic flows, Q. Appl. Math. 9 (1952) 421-423.

[31] J.C. Maxwell, The scientific papers of James Clerk Maxwell, Cambridge University Press, 1890.

[32] I. Müller, Theory of mixtures of fluids, Arch. Rat. Mech. Anal., 28 (1968) 1-38.

[33] I. Müller, Thermodynamics, Interaction of Mechanics and Mathematics Series, Pitman, London, 1985.

[34] I. Müller, T. Ruggeri, Rational Extended Thermodynamics, Springer-Verlag, New York, 1998.

[35] S. Ono, S. Kondo, Molecular theory of surface tension in liquid in "Structure of liquids", S. Flügge (ed.) Encyclopedia of Physics, X, Springer, Berlin, 1960.

[36] Y. Rocard, Thermodynamique, Masson, Paris, 1967.

[37] J.S. Rowlinson, B. Widom, Molecular theory of capillarity, Clarendon Press, Oxford, 1984.

[38] J.S. Rowlinson, F.L. Swinton, Liquid and liquid mixtures, Butterworths, Wolborn MA, 1982.

[39] R. Sampaio, W.O. Williams, On the viscosities of liquid mixtures, J. Appl. Math. and Physics, 28, (1977) 607-613.

[40] J. Serrin, Mathematical principles of classical fluid mechanics, Encyclopedia of Physics, VIII/1, Springer, Berlin, (1959) pp. 144-150.

[41] M. Slemrod, Admissibility criteria for propagating phase boundaries in a van der Waals fluid, Arch. Rat. Mech. Anal., 83 (1983) 301-313.

[42] J.D. van der Waals, Thermodynamique de la capillarité dans l'hypothèse d'une variation continue de densité, Archives Néerlandaises 28 (1894-1895) 121-209.

[43] G.B. Whitham, Linear and nonlinear waves, Wiley, New-York, 1974.

[44] B. Widom, What do we know that van der Waals did not know?, Physica A 263 (1999) 500-515. 